\title{
SARS-CoV-2 Infection Hospitalization Rate and Infection Fatality Rate among the Non- \\ Congregant Population in Connecticut
}

Shiwani Mahajan, MBBS, MHS; ${ }^{1,2}$ César Caraballo, MD; ${ }^{1,2}$ Shu-Xia Li, PhD; ${ }^{1}$

Claire Dong, MPH; ${ }^{1}$ Lian Chen, MS $;{ }^{1}$ Sara K. Huston, BS $;{ }^{3}$ Rajesh Srinivasan, PhD; ${ }^{3}$ Carrie A. Redlich, MD, MPH $;{ }^{4}$ Albert I. Ko, MD $;{ }^{5}$ Jeremy S. Faust, MD, MD $;{ }^{6}$ Howard P. Forman, MD, MBA; ${ }^{7,8}$ Harlan M. Krumholz, MD, SM ${ }^{1,2,8}$

${ }^{1}$ Center for Outcomes Research and Evaluation, Yale-New Haven Hospital, New Haven, CT

${ }^{2}$ Section of Cardiovascular Medicine, Department of Internal Medicine, Yale School of

Medicine, New Haven, CT

${ }^{3}$ The Gallup Organization, Washington, DC

${ }^{4}$ Yale Occupational and Environmental Medicine Program, Department of Internal Medicine, Yale School of Medicine, New Haven, CT

${ }^{5}$ Department of Epidemiology of Microbial Diseases, Yale School of Public Health, New Haven, CT

${ }^{6}$ Department of Emergency Medicine, Division of Health Policy and Public Policy, Brigham and Women's Hospital, Harvard Medical School, Boston, MA

${ }^{7}$ Department of Radiology and Biomedical Imaging, Yale School of Medicine, New Haven, CT

${ }^{8}$ Department of Health Policy and Management, Yale School of Public Health, New Haven, CT

\section{Address for correspondence:}

Harlan M. Krumholz MD, SM

1 Church Street, Suite 200, New Haven, CT 06510 USA 
medRxiv preprint doi: https://doi.org/10.1101/2020.10.30.20223461; this version posted November 4, 2020. The copyright holder for this preprint (which was not certified by peer review) is the author/funder, who has granted medRxiv a license to display the preprint in perpetuity. It is made available under a CC-BY-NC-ND 4.0 International license .

Telephone: 203-764-5885; Fax: 203-764-5653

Email: harlan.krumholz@yale.edu; Twitter: @ hmkyale

Word count: 1233 


\section{ABSTRACT}

Importance: COVID-19 case fatality and hospitalization rates, calculated using the number of confirmed cases of COVID-19, have been described widely in the literature. However, the number of infections confirmed by testing underestimates the total infections as it is biased based on the availability of testing and because asymptomatic individuals may remain untested. The infection fatality rate (IFR) and infection hospitalization rate (IHR), calculated using the estimated total infections based on a representative sample of a population, is a better metric to assess the actual toll of the disease.

Objective: To determine the IHR and IFR for COVID-19 using the statewide SARS-CoV-2 seroprevalence estimates for the non-congregate population in Connecticut.

Design: Cross-sectional.

Setting: Adults residing in a non-congregate setting in Connecticut between March 1 and June 1, 2020.

Participants: Individuals aged 18 years or above.

Exposure: Estimated number of adults with SARS-CoV-2 antibodies.

Main Outcome and Measures: COVID-19-related hospitalizations and deaths among adults residing in a non-congregate setting in Connecticut between March 1 and June 1, 2020.

Results: Of the 2.8 million individuals residing in the non-congregate settings in Connecticut through June 2020, 113,515 (90\% CI 56,758-170,273) individuals had SARS-CoV-2 antibodies. There were a total of 9425 COVID-19-related hospitalizations and 4071 COVID-19-related deaths in Connecticut between March 1 and June 1, 2020, of which 7792 hospitalizations and 1079 deaths occurred among the non-congregate population. The overall COVID-19 IHR and IFR was $6.86 \%(90 \% \mathrm{CI}, 4.58 \%-13.72 \%)$ and $0.95 \%(90 \% \mathrm{CI}, 0.63 \%-1.90 \%)$ among the non- 
congregate population. Older individuals, men, non-Hispanic Black individuals and those belonging to New Haven and Litchfield counties had a higher burden of hospitalization and deaths, compared with younger individuals, women, non-Hispanic White or Hispanic individuals, and those belonging to New London county, respectively.

Conclusion and Relevance: Using representative seroprevalence estimates, the overall COVID19 IHR and IFR were estimated to be $6.86 \%$ and $0.95 \%$ among the non-congregate population in Connecticut. Accurate estimation of IHR and IFR among community residents is important to guide public health strategies during an infectious disease outbreak. 


\section{BACKGROUND}

Accurate estimation of the hospitalization and fatality rate is important to guide public health strategies during infectious disease outbreaks. Although the case fatality rate (CFR), defined as the proportion of deaths of the confirmed cases, is a commonly used metric, it will be biased based on the availability of testing, especially early in the outbreak. ${ }^{1}$ Moreover, since COVID-19 symptoms range widely, mild or asymptomatic infections may be untested. Thus, the number of infections confirmed by testing will underestimate the total infections, inflating the estimated fatality rate.

A better estimate of the actual toll is the infection fatality rate (IFR), defined as the proportion of deaths of the total infected individuals, in which the denominator is ideally based on a representative sample of a population. For hospitalizations, the infection hospitalization rate (IHR) is a comparable measure. Accordingly, using results of the recently conducted statewide SARS-CoV-2 seroprevalence study — the Post-Infection Prevalence study (PIP)—in Connecticut, ${ }^{2}$ we assessed the SARS-CoV-2 IHR and IFR.

\section{METHODS}

Based on the PIP study, ${ }^{2}$ the seroprevalence of SARS-CoV-2 antibodies was $4.0 \%(90 \%$ CI $2.0 \%-6.0 \%$ ) among a representative population of adults residing in non-congregate settings in Connecticut before June 2020. We used this estimate to calculate the overall population estimates for individuals infected with SARS-CoV-2 using the 2018 American Community Survey. We also estimated the number of individuals with SARS-CoV-2 antibodies by age, sex, race/ethnicity, and region. 
Information on the total COVID-19-related hospitalizations and deaths among the noncongregate population in Connecticut, between March 1 and June 1, 2020, was provided by the Connecticut Hospital Association and the Connecticut Department of Public Health, respectively. The diagnostic codes used to identify COVID-19-related hospitalizations are listed in eTable 1. Total COVID-19 deaths included both confirmed and probable COVID-19 deaths (details in eMethods).

IHR and IFR were defined as the number of individuals who were hospitalized and died, respectively, due to COVID-19 divided by the total estimated number of individuals who had COVID-19 using the seroprevalence estimates. The margin of error for our estimates were calculated at the $90 \%$ confidence level in accordance with the design of the PIP study, however, estimates at $95 \%$ CI have also been provided. Due to sample size limitations, the upper end of the confidence interval was non-estimable (NE) when stratifying by some of the sociodemographic characteristics. All statistical analyses were performed using R version 4.0.2.

\section{RESULTS}

Of the 2.8 million individuals residing in the non-congregate settings in Connecticut, 113,515 (90\% CI 56,758-170,273) had SARS-CoV-2 antibodies (eTable 2). Between March 1 and June 1, 2020, there were a total of 9425 COVID-19-related hospitalizations and 4071 COVID-19-related deaths in Connecticut, of which 7792 hospitalizations and 1079 deaths occurred among the non-congregate population.

The overall COVID-19 IHR and IFR was 6.86\% (90\% CI, 4.58\%-13.72\%) and $0.95 \%$ (90\% CI, 0.63\%-1.90\%) among the non-congregate population (Table 1). When compared with women, men had a higher IHR (12.21\% [90\% CI, 6.23\%-NE] vs 4.64\% [90\% CI, 3.00\%- 
$10.25 \%])$ and IFR (2.39\% [90\% CI, $1.22 \%-59.71 \%]$ vs $0.34 \%$ [90\% CI, $0.22 \%-0.75 \%])$. By age, IHR and IFR ranged from $0.80 \%(90 \% \mathrm{CI}, 0.36 \%-\mathrm{NE})$ and $0.01 \%(90 \% \mathrm{CI}, 0.01 \%-\mathrm{NE})$ among individuals aged $18-29$ years, to $12.43 \%(90 \% \mathrm{CI}, 6.46 \%-\mathrm{NE})$ and $1.30 \%(90 \% \mathrm{CI}$, 0.68\%-16.94\%) among those aged 55-64 years. By race/ethnicity, IHR and IFR were significantly higher among Black individuals $(9.90 \%$ [90\% CI, 5.32\%-70.40\%] and 1.46\% [90\% CI, $0.78 \%-10.35 \%])$, as compared with White (7.42\% [90\% CI, $4.56 \%-20.05 \%]$ and $1.12 \%$ [ $90 \%$ CI, $0.68 \%-3.01 \%]$ ) and Hispanic (1.04\% [90\% CI, 0.78\%-1.57\%] and 0.23\% [90\% CI, $0.17 \%-0.35 \%])$ individuals. New Haven and Litchfield counties had the highest IHR $(9.04 \%$ [90\% CI, 4.65\%-NE]) and IFR (1.65\% [90\% CI, 0.55\%-NE]), respectively, whereas New London county had the lowest IHR (4.04\% [90\% CI, 1.37\%-NE]) and IFR (0.57\% [90\% CI, 0.19\%-NE]). IHR and IFR estimates with $95 \% \mathrm{CI}$ are presented in eTable 3 . The confirmed and probable IFRs are shown separately in eTable 4.

\section{DISCUSSION}

Using seroprevalence estimates, we found that, through June 2020, Connecticut's COVID-19 IHR and IFR were $6.86 \%$ and $0.95 \%$, respectively. The rates of hospitalization and death varied and older individuals, men, non-Hispanic Black individuals, and those belonging to New Haven and Litchfield counties had the highest burden of adverse outcomes. Our estimates are distinctive because they reflect people living in the community and are based on a methodology that sought to obtain a representative estimate for the denominator.

There has been continued controversy about the IFR and the literature is replete with

widely varied estimates. ${ }^{3-7}$ A recent meta-analysis on COVID-19 IFR, estimated a global pooled COVID-19 IFR of $0.68 \%,{ }^{5}$ with values ranging from $0.01 \%$ to $1.60 \%$. However, the studies 
included did not have a representative sample or separate out special populations, such as those in nursing homes. Moreover, IFR is not an inherent characteristic of the disease, but rather a confluence of the pathogen virulence, sociodemographic and clinical characteristics of the population, health care availability and quality, therapeutic availability, and accurate counting and reporting of COVID-19-related deaths. As such, an overall IFR may not be very informative given the heterogeneity among subgroups.

The COVID-19 IHR is not well described and most studies report the CHR, which also varies widely in the literature. ${ }^{8-10}$ The CDC estimated a US COVID-19 CHR of $14.0 \%$ for infections before June 2020. ${ }^{10}$ As expected, this CHR estimate was higher than Connecticut's estimated IHR in our study (6.9\%), as IHR includes the total estimated infections rather than detected positive cases only. Moreover, our estimate excluded individuals from congregate settings, which had a higher burden of adverse outcomes.

Our subgroup findings are notable. Although it has been noted that age and sex are associated with disease severity, prior studies have been hampered by including nursing home residents and biased by testing patterns. We had the opportunity to identify hospitalizations and deaths among the non-congregate population in Connecticut and show that, even in the community, these associations remain. Of note, Black individuals had a higher IFR and IHR than White individuals, though the difference was not statistically significant. Prior studies have shown that Black individuals have had disproportionately higher infection rates, even as some studies indicate that hospital mortality does not vary by race/ethnicity. ${ }^{11}$ Our findings highlight that the burden of COVID-19 among Black subpopulations is not just about infection rates but also worse outcomes. The IHR for Hispanic individuals was lower than for White and Black individuals, which was in accordance with previously reported low hospital admission rates 
among Hispanic individuals testing positive for SARS-CoV-2 in Baltimore-Washington, DC ${ }^{12}$ and may be associated with the lower insurance rates among the Hispanic subpopulation in Connecticut. $^{13}$

Our study has some limitations. First, total COVID-19-related hospitalizations could have been underestimated due to limited testing availability, underestimating the IHR estimate. Second, although antibodies are specific indicators of past SARS-CoV-2 infection, their concentration may decrease few months after exposure ${ }^{14}$ and there are differences in the sensitivity of available assays. ${ }^{15}$ Hence, the total infections may be biased lower, overestimating our estimates. Nevertheless, representative seroprevalence studies provide important information regarding infections in a community and can provide robust estimates of the IHR and IFR, when combined with hospitalization and death data.

In conclusion, using representative seroprevalence estimates, we estimate an IHR and IFR of $6.86 \%$ (90\% CI 4.58\%-13.72\%) and $0.95 \%$ (90\% CI 0.63\%-1.90\%), respectively, for COVID-19 infections through June 1, 2020, among the non-congregate population in Connecticut. 
ACKNOWLEDGEMENTS: The authors thank the Connecticut Department of Public Health and the Connecticut Hospital Association for their support.

SOURCES OF FUNDING: This project was supported by the Centers for Disease Control and Prevention through the CARES Act.

CONFLICTS OF INTEREST: Dr. Ko reports grants from Bristol Myer Squib Foundation, Regeneron, and Serimmune, and honoraria from Bristol Myer Squib, outside the submitted work. In the past three years, Dr. Krumholz received expenses and/or personal fees from UnitedHealth, IBM Watson Health, Element Science, Aetna, Facebook, the Siegfried and Jensen Law Firm, Arnold and Porter Law Firm, Martin/Baughman Law Firm, F-Prime, and the National Center for Cardiovascular Diseases in Beijing. He is an owner of Refactor Health and HugoHealth, and had grants and/or contracts from the Centers for Medicare \& Medicaid Services, Medtronic, the U.S. Food and Drug Administration, Johnson \& Johnson, and the Shenzhen Center for Health Information. The other co-authors report no potential competing interests. 


\section{REFERENCES}

1. Niforatos JD, Melnick ER, Faust JS. Covid-19 fatality is likely overestimated. BMJ. 2020;368:m1113.

2. Mahajan S, Srinivasan R, Redlich CA, et al. Seroprevalence of SARS-CoV-2-specific IgG antibodies among adults living in Connecticut: Post-Infection Prevalence (PIP) study. medRxiv. 2020:2020.2008.2004.20168203.

3. Blackburn J, Yiannoutsos CT, Carroll AE, Halverson PK, Menachemi N. Infection fatality ratios for COVID-19 among noninstitutionalized persons 12 and older: results of a random-sample prevalence study. Ann Intern Med. 2020:M20-5352.

4. Erikstrup C, Hother CE, Pedersen OBV, et al. Estimation of SARS-CoV-2 infection fatality rate by real-time antibody screening of blood donors. Clin Infect Dis. 2020.

5. Meyerowitz-Katz G, Merone L. A systematic review and meta-analysis of published research data on COVID-19 infection-fatality rates. medRxiv. 2020:2020.2005.2003.20089854.

6. Pastor-Barriuso R, Perez-Gomez B, Hernan MA, et al. SARS-CoV-2 infection fatality risk in a nationwide seroepidemiological study. medRxiv. 2020:2020.2008.2006.20169722.

7. Russell TW, Hellewell J, Jarvis CI, et al. Estimating the infection and case fatality ratio for coronavirus disease (COVID-19) using age-adjusted data from the outbreak on the Diamond Princess cruise ship, February 2020. Euro Surveill. 2020;25(12).

8. Price-Haywood EG, Burton J, Fort D, Seoane L. Hospitalization and mortality among Black patients and White patients with Covid-19. N Engl J Med. 2020;382(26):25342543. 
9. Mendy A, Apewokin S, Wells AA, Morrow AL. Factors associated with hospitalization and disease severity in a racially and ethnically diverse population of COVID-19 patients. medRxiv. 2020:2020.2006.2025.20137323.

10. Stokes EK, Zambrano LD, Anderson KN, et al. Coronavirus disease 2019 case surveillance-United States, January 22-May 30, 2020. MMWR Morb Mortal Wkly Rep. 2020;69(24):759.

11. McPadden J, Warner F, Young HP, et al. Clinical Characteristics and outcomes for 7,995 patients with SARS-CoV-2 infection. medRxiv. 2020:2020.2007.2019.20157305.

12. Martinez DA, Hinson JS, Klein EY, et al. SARS-CoV-2 positivity rate for Latinos in the Baltimore-Washington, DC region. JAMA. 2020;324(4):392-395.

13. Becker AL. Health disparities in Connecticut: causes, effects, and what we can do. Connecticut Health Foundation. https://www.cthealth.org/publication/health-disparitiesin-connecticut-causes-effects-and-what-we-can-do/. Accessed October 24, 2020.

14. Ibarrondo FJ, Fulcher JA, Goodman-Meza D, et al. Rapid decay of anti-SARS-CoV-2 antibodies in persons with mild Covid-19. N Engl J Med. 2020.

15. Lisboa Bastos M, Tavaziva G, Abidi SK, et al. Diagnostic accuracy of serological tests for covid-19: systematic review and meta-analysis. BMJ. 2020;370:m2516-m2516. 
Table 1. Infection hospitalization rate and infection fatality rate among the non-congregate population in Connecticut between March 1 and June 1, 2020, by sociodemographic characteristics.

\begin{tabular}{|c|c|c|c|c|}
\hline & $\begin{array}{l}\text { COVID-19-related } \\
\text { Hospitalizations, N }\end{array}$ & $\begin{array}{c}\text { Infection Hospitalization } \\
\text { Rate, Weighted \% (90\% CI) }\end{array}$ & $\begin{array}{l}\text { Total* COVID-19- } \\
\text { related Deaths, N }\end{array}$ & $\begin{array}{c}\text { Total Infection Fatality } \\
\text { Rate, Weighted \% (90\% CI) }\end{array}$ \\
\hline State Total & 7792 & $6.86(4.58-13.72)$ & 1079 & $0.95(0.63-1.90)$ \\
\hline \multicolumn{5}{|l|}{ Sex } \\
\hline Men & 4166 & $12.21(6.23-\mathrm{NE})$ & 815 & $2.39(1.22-59.71)$ \\
\hline Women & 3625 & $4.64(3.00-10.25)$ & 264 & $0.34(0.22-0.75)$ \\
\hline \multicolumn{5}{|l|}{ Age-group } \\
\hline $18-29$ & 288 & $0.80(0.36-\mathrm{NE})$ & 5 & $0.01(0.01-\mathrm{NE})$ \\
\hline $30-44$ & 855 & $2.68(1.38-43.85)$ & 34 & $0.11(0.06-1.74)$ \\
\hline $45-54$ & 1013 & $3.09(1.81-10.74)$ & 59 & $0.18(0.11-0.63)$ \\
\hline $55-64$ & 1660 & $12.43(6.46-\mathrm{NE})$ & 174 & $1.30(0.68-16.94)$ \\
\hline$\geq 65$ & 3918 & 79.89 (31.96-NE) & 807 & $16.46(6.58-\mathrm{NE})$ \\
\hline \multicolumn{5}{|l|}{ Race/Ethnicity } \\
\hline Non-Hispanic Black & 1762 & $9.90(5.32-70.40)$ & 259 & $1.46(0.78-10.35)$ \\
\hline Non-Hispanic White & 3948 & $7.42(4.56-20.05)$ & 593 & $1.12(0.68-3.01)$ \\
\hline Hispanic & 845 & $1.04(0.78-1.57)$ & 190 & $0.23(0.17-0.35)$ \\
\hline \multicolumn{5}{|l|}{ County } \\
\hline New Haven & 2101 & $9.04(4.65-\mathrm{NE})$ & 270 & $1.16(0.60-19.74)$ \\
\hline New London & 148 & $4.04(1.37-\mathrm{NE})$ & 21 & $0.57(0.19-\mathrm{NE})$ \\
\hline Middlesex & 231 & $\mathrm{NE}$ & 32 & $\mathrm{NE}$ \\
\hline Fairfield & 2665 & $6.39(3.28-\mathrm{NE})$ & 399 & $0.96(0.49-18.16)$ \\
\hline Hartford & 1755 & $6.21(3.27-62.08)$ & 294 & $1.04(0.55-10.40)$ \\
\hline Litchfield & 195 & $8.26(2.75-\mathrm{NE})$ & 39 & 1.65 (0.55-NE) \\
\hline
\end{tabular}

